\section{1-S06.05 EXPLORING THE POTENTIAL IMPACT ON HIV INCIDENCE OF A REDUCTION IN CONCURRENCY IN RURAL UGANDA: A MODELLING STUDY}

doi:10.1136/sextrans-2011-050109.35

${ }^{1} \mathrm{~N}$ McCreesh, ${ }^{2} \mathrm{~K}$ O'Brien, ${ }^{1} \mathrm{R}$ Nsubuga, ${ }^{1} \mathrm{~L}$ A Shafer, ${ }^{3} \mathrm{R}$ Bakker, ${ }^{1} \mathrm{~J}$ Seeley, ${ }^{2} \mathrm{~W}$ Richard. ${ }^{1}$ MRC/UVRI Uganda Research Unit on AIDS, Entebbe, Uganda; ${ }^{2}$ London School of Hygiene and Tropical Medicine, UK; ${ }^{3}$ Erasmus MC, University Medical Center, Rotterdam, Netherlands

Background The empirical evidence for an association between sexual partnership concurrency and increased HIV acquisition or transmission is equivocal, and the likely impact of changes in the prevalence of concurrency on HIV incidence in countries with generalised HIV epidemics has not been explored. Despite this, a number of SSA counties have planned or implemented campaigns against concurrency. Evaluation of these campaigns is unlikely to provide strong evidence for the likely impact of changes in concurrency on HIV incidence as it will not be possible to separate the effects of a reduction in concurrency from the effects of a reduction in partnership incidence. This modelling study investigates the potential effect on HIV incidence of an intervention to reduce concurrency in rural Uganda, and in other sub-Saharan Africa populations with higher levels of concurrency.

Methods Data on the demography, sexual behaviour, and HIV prevalence of a cohort in Masaka, Uganda were used to parameterise an individual-based HIV transmission model. The UNAIDS recommended definition of concurrency was used. Four scenarios with different prevalences of male and female concurrency were modelled. An intervention that reduced partnership concurrency by $50 \%$ between 2010 and 2020 (keeping overall partnership incidence constant) was introduced and the impact on HIV incidence in 2020 was calculated.

Results $9.6 \%(7.9 \%-11.4 \%)$ of men and $0.2 \%(0.0 \%-0.4 \%)$ of women reported concurrent sexual partnerships in rural Uganda in 2010. Preliminary results suggest that in the model scenario simulating this reported behaviour, the intervention reduces the incidence of HIV by $7.4 \%$ in men and $19.9 \%$ in women (Abstract O1S06.05 figure 1, scenario 1a). If more female concurrency is simulated, the potential reductions of the intervention are greater $(11.1 \%-14.8 \%$ in men and $21.6 \%-24.7 \%$ in women, Abstract $01-$ S06.05 figure 1, scenarios $2 \mathrm{a}$ and $3 \mathrm{a}$ ). The potential reductions are also greater when a higher prevalence of male concurrency is simulated $(9.7 \%-15.5 \%$ in men and $26.6 \%-29.6 \%$ in women, Abstract O1-S06.05 figure 1, scenarios $1 b, 2 b$ and $3 b$ ).

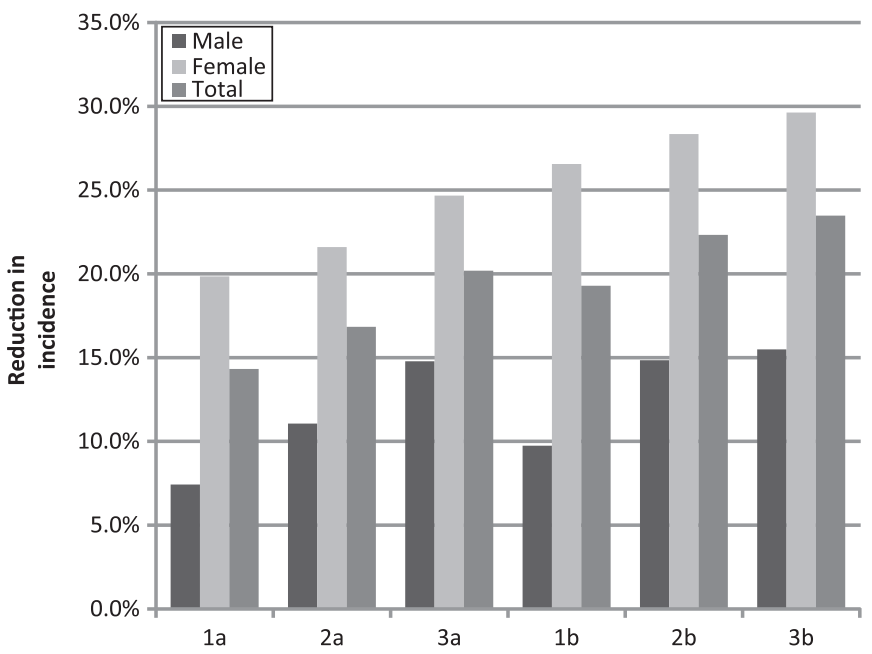

Abstract 01-S06.05 Figure 1 The impact of the intervention on HIV incidence in 2020 .
Conclusions In this setting, interventions against concurrency have the potential to reduce the HIV incidence and may have a higher impact on the incidence of HIV in women than in men. However a large simulated change in behaviour resulted in only a moderate decrease in HIV incidence. This study does not provide strong support for the prioritisation of concurrency as a target for behaviour change interventions.

\section{1-S06.06 PATTERNS AND TRENDS IN CONCURRENCY AND POLYGAMY IN RURAL ZIMBABWE, 1998-2008}

doi:10.1136/sextrans-2011-050109.36

${ }^{1} \mathrm{~F}$ Takavarasha, ${ }^{2} \mathrm{~S}$ Gregson, ${ }^{2} \mathrm{~J}$ Eaton, ${ }^{2} \mathrm{C}$ Schumacher, ${ }^{3 \mathrm{P}}$ Mushati, ${ }^{2} \mathrm{G}$ Garnett, ${ }^{3} \mathrm{C}$ Nyamukapa. ${ }^{1}$ Biomedical Research and Training Institute, Zimbabwe Harare, Zimbabwe; ${ }^{2}$ School of Public Health, Imperial College London, Harare, UK; ${ }^{3}$ Biomedical Research and Training Institute, Harare, Zimbabwe

Background It has been suggested that the decline in HIV prevalence in Zimbabwe from the late 1990s may be partly due to reductions in sexual concurrency but little is known about levels and trends in the different forms of concurrency or their association with HIV risk.

Methods We use data from four rounds (1998-2000, 2001-03, 2003-05, 2006-08) of a large longitudinal population-based HIV survey to investigate patterns of non-spousal (two or more current sexual partners, at least one of whom is not a spouse) and spousal (multiple spouses but currently no extra-marital sexual partners) concurrency, and associations with prevalent HIV infection in rural east Zimbabwe.

Results $15.4 \%$ (95\% CI $14.4 \%$ to $16.5 \%$ ) of men (17-54 yrs, $\mathrm{N}=4327)$ and $1.9 \%$ (1.5\% to $2.3 \%$ ) of women (15-44 yrs, $\mathrm{N}=5148)$ reported concurrent sexual partners at baseline. $11.7 \%(10.8 \%$ to $12.7 \%$ ) and $3.7 \%$ (3.2\% to $4.3 \%$ ) of men reported non-spousal and spousal concurrency, respectively. Non-spousal concurrency was most common in single $(16 \%)$ and divorced/widowed (11\%) men but was also reported by married men with single $(6.5 \%)$ and multiple (4\%) spouses (Abstract O1-S06.06 figure 1). HIV prevalence is similar in men with (19.2\%) and without (19.5\%) concurrent partners but is higher in those with concurrent partners after controlling for age (age-adj. OR (aOR), 1.37; $\mathrm{p}=0.02)$. However, after excluding non-sexually experienced men, this difference was not statistically significant ( $\mathrm{aOR}, 1.27, \mathrm{p}=0.08)$. Among all men, HIV prevalence was higher for those with non-spousal concurrency (aOR, 1.38; $\mathrm{p}=0.015$ ) but not for those with spousal concurrency (aOR, 1.26; $\mathrm{p}=0.2$ ). For women, concurrency (all non-spousal) was $1.9 \%(95 \%$ CI $1.2 \%$ to $2.7 \%)$ in single women, $0.4 \%(0.2 \%$ to $0.7 \%)$

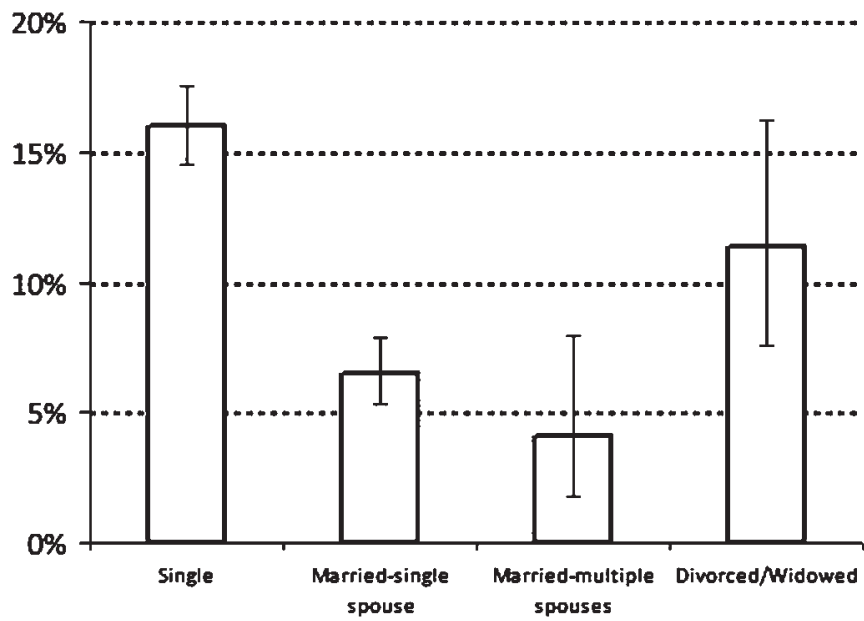

Abstract 01-S06.06 Figure 1 Prevalence of non-spousal concurrency by marital status, Men, $17-54$ years. 